\title{
Modelo de comportamiento estructural de muros entramados de madera en el siglo XIX en España
}

\author{
Structural behavior model of timber-framed walls in the nineteenth century \\ in Spain
}

J. Santa Cruz-Astorqui ${ }^{(*)}$, M. Del Río(*)

RESUMEN

En este artículo se analiza el comportamiento estructural del muro entramado de madera. Muro compuesto de una retícula de piezas verticales (pies derechos) y horizontales (carreras), cuyos cuarteles se rellenan normalmente con yesones. Este sistema es ampliamente utilizado como muro portante en la edificación residencial española entre los siglos XVII y XIX. Del análisis, se concluye que las cargas se transmiten principalmente a través de los rellenos por compresión vertical, y que la colaboración de las carreras por flexión es despreciable, salvo en aquellos casos en los que no exista el relleno del cuartel inferior. Además, mediante la simulación numérica por elementos finitos, se obtiene una expresión que determina el porcentaje de carga total que absorben los pies derechos y los rellenos en función de sus rigideces a compresión, con el objeto de cuantificar el estado tensional de los mismos, aspecto fundamental en el diseño y dimensionamiento de refuerzos en rehabilitación estructural.

Palabras clave: Rehabilitación estructural; muro de entramado de madera; análisis por elementos finitos.

\section{ABSTRACT}

In this article the structural behavior of a timber-framed wall is analysed. The wall is composed by a timber grid of vertical parts (posts) and horizontal elements (beams), whose panels are normally filled with plaster rubble. This system was widely used as a load-bearing wall system in the Spanish residential buildings between the 17th and 19th centuries. The research concludes that the loads are transmitted mainly through the infills by vertical compression, and that the deformation of the beams by bending is negligible, except in those cases in which there is no infill at the bottom. Also, using numerical simulation by finite elements, a formula determining the percentage of total loads absorbed by posts and infills is obtained depending on their rigidity to compression, so as to quantify the stress state of them. This is a fundamental aspect in the design and dimensioning of reinforcements in structural rehabilitation.

Keywords: Structural rehabilitation; timber-framed wall; finite element analysis.

(*) Universidad Politécnica de Madrid, (España).

Persona de contacto/Corresponding author: jaime.santacruz@upm.es (J. Santa Cruz-Astorqui)

Cómo citar este artículo/Citation: Santa Cruz-Astorqui, J. (2014). Modelo de comportamiento estructural de muros entramados de madera en el siglo XIX en España. Informes de la Construcción, 66(536): e048, doi: http://dx.doi.org/10.3989/ic.14.030.

Licencia/License: Salvo indicación contraria, todos los contenidos de la edición electrónica de Informes de la Construcción se distribuyen bajo una licencia de uso y distribución Creative Commons Reconocimiento no Comercial 3.o. España (cc-by-nc). 


\section{INTRODUCCIÓN}

El muro entramado de madera es característico de la edificación residencial española entre los siglos XVII y XIX. En contraste con los muros de fábrica de ladrillo o de mampostería de piedra (mas propios en la arquitectura civil y religiosa), el muro entramado constituye una solución de muro portante más ligera y económica, y también de más rápida ejecución, aunque de menor durabilidad y resistencias mecánica y ante el fuego (1) (2).

Este tipo de muro está formado por una retícula de piezas de madera que se rellenan posteriormente con yesones, cascotes de derribo, adobes, tapial, y en ocasiones, fábrica de ladrillo. Dicha retícula se compone de pies derechos de madera cada $1,40-1,80 \mathrm{~m}$ que soportan vigas o carreras que sirven de apoyo a los forjados, según se indica en la Figura 1.

En Madrid, la construcción residencial entre los siglos XVI y XIX se realizó mayoritariamente con este tipo de muros tanto en muros interiores como en muros de fachada a los patios interiores, siendo los muros de fachada a la calle construidos con fábrica de ladrillo. En la configuración de este tipo de muros, representada en la Figura 1, el pie derecho apoya directamente sobre la carrera inferior o sobre las viguetas de forjado.

La unión entre los pies derechos/vigas de madera y el relleno de yesones, se soluciona tradicionalmente mediante tomiza (cuerda de esparto) enrollada al pie derecho o la viga, y que actúa como conector entre madera y yeso. Esta solución también se aplica a las viguetas de forjado, para asegurar su unión con el relleno del entrevigado.

En cuanto a su funcionamiento estructural, podríamos clasificar el muro entramado como muro compuesto, dado que tanto el entramado de madera como el relleno de los cuarteles, contribuyen de forma combinada a su respuesta estructural.

Si bien la bibliografía sobre cerramientos entramados ligeros de madera es abundante, no se han encontrado referencias al comportamiento estructural de los muros tradicionales de entramado pesado de madera, salvo el caso de las Pombalinas de Lisboa y otros casos, cuyos muros entramados con crucetas han sido ampliamente estudiados por varios autores, como Cardoso et al. (4), Mendes et al. (5) y Ramos et al. (6), que aportan modelos que explican el funcionamiento de este tipo de estructuras, orientados al proyecto de refuerzos ante sismo.
Existe la idea generalizada de que en este tipo de muros, las cargas gravitatorias son asumidas únicamente por lo pies derechos, que reciben las cargas de los forjados a través de la flexión de las carreras, y que la función de los rellenos es únicamente la de cegar el cerramiento. Pocos son los autores que defienden lo contario, como Biehholdt et al. (7) que plantean un modelo de funcionamiento estructural en el que los pies derechos actúan como refuerzo ante el pandeo del muro y los rellenos colaboran con la capacidad de carga total del mismo. Doudoumis (8) aporta un modelo mediante elementos finitos que explica el funcionamiento compuesto de los muros entramados de madera-fábrica, estudiando el comportamiento no lineal de la interfase relleno-madera, describiendo la colaboración entre los dos componentes.

Entre las ventajas de la utilización de los muros entramados frente a los muros de fábrica homogéneos, está su menor peso. Los muros de fábrica homogéneos, requieren de un gran espesor para evitar el colapso por flexión, dado que los morteros de junta no son capaces de resistir las tensiones de tracción provocadas por dicha flexión. En el caso de los muros entramados de madera, los pies derechos actúan como rigidizadores ante las deformaciones provocadas por la flexión, permitiendo así que el muro no pandee a pesar de su gran esbeltez. El fenómeno de flexión descrito se refiere a los momentos inducidos por la posible excentricidad de la carga de los forjados respecto al plano medio del muro (p.ej. en muros de fachada o medianeros) y/o por las irregularidades geométricas propias del muro (desplomes, desplazamientos, etc).

Se puede considerar a este tipo de muros como los precursores del muro de bloque armado, en el que los nervios armados resisten los esfuerzos derivados de las posibles flexiones, pero es el conjunto del muro el que absorbe las cargas gravitatorias.

El material de relleno de los cuarteles posee una rigidez y resistencia a la compresión suficientes como para absorber las tensiones generadas por las cargas de los forjados y el peso propio del muro. De igual forma, las acciones horizontales en el plano del muro, quedan estabilizadas por la combinación de esfuerzos de las tornapuntas y las bielas de compresión que se forman en los rellenos.

En un muro entramado, el porcentaje de carga que absorben los pies derechos y los rellenos, depende de la relación entre sus rigideces a compresión. Las cargas gravitatorias se transmiten hasta la cimentación principalmente a través de los rellenos por compresión vertical, atravesando las carreras que se comportan como meras soleras de apoyo de los forjados.

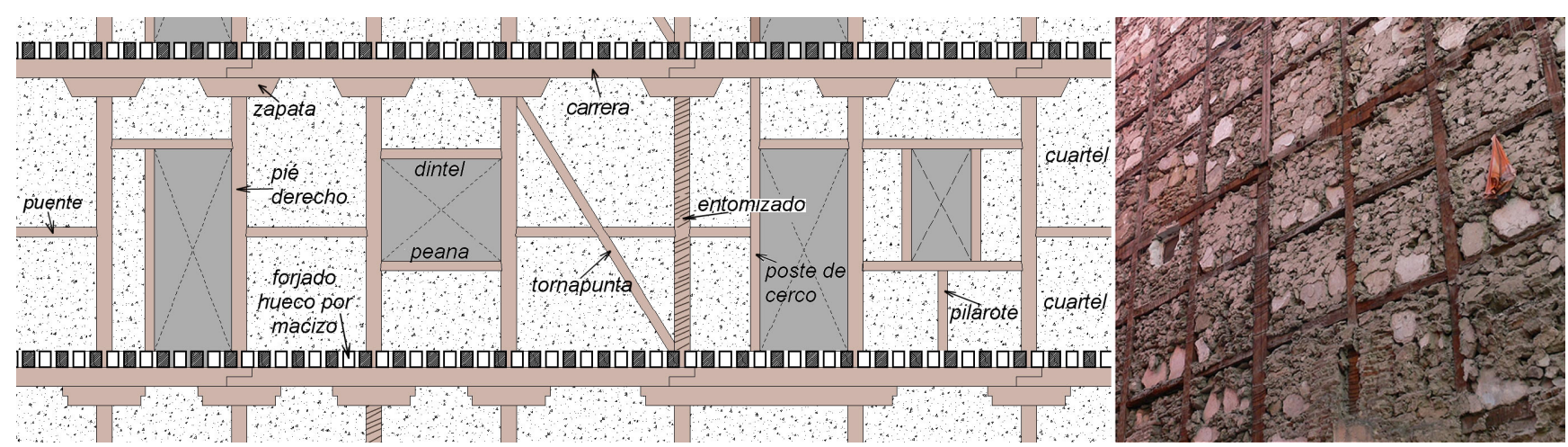

Figura 1. Componentes de un muro entramado típico madrileño (3). 
Parte de las cargas de los rellenos se transfieren a los pies derechos en cada planta, siguiendo un mecanismo de arco de descarga (3).

\section{OBJETIVOS Y METODOLOGÍA}

El objetivo principal de esta investigación es la propuesta de un modelo que explique el mecanismo de funcionamiento estructural de este tipo de muros, y la obtención de una expresión analítica que permita cuantificar el estado tensional de los pies derechos y rellenos en cada planta, fundamental para diseñar cualquier intervención en la rehabilitación estructural de estos muros.

Para expresar mejor el modelo propuesto, se recurre a la simulación numérica por el método de elementos finitos de un muro entramado tipo de cuatro plantas, sometido a su peso propio y a la carga lineal de los forjados que apoyan en él. Se realizan simulaciones de muros ciegos y de muros con ausencia total o parcial de rellenos, para confirmar el modelo propuesto. La utilización del análisis por elementos finitos ha sido ampliamente descrita por varios autores en el estudio de muros entramados de madera (4) (5) (6) (7) (8) y (9).

Para el mallado del modelo se han utilizado elementos tipo «Shell» de 4 nodos. Para la simulación de las uniones entre piezas de madera, así como entre madera y rellenos, se han utilizado elementos especiales de contacto (solo compresión), con un coeficiente de rozamiento de o,5 -Doudoumis (8)- que representa de forma aproximada la adherencia que proporciona la tomiza que envuelve los pies derechos, y los clavos que normalmente fijan las piezas de madera entre sí. En consecuencia se prevé un cierto deslizamiento entre las partes contactadas, pero el rozamiento existente es suficiente como para producir una cierta transferencia tangencial de carga entre rellenos y pies derechos. Debido a la no-lienalidad de los elementos de contacto, se ha utilizado un solver nonlinear static (Straus7/Strand7), utilizando para ello 10 escalones de carga (de $10 \%$ a $100 \%$ ).

Los datos de los diferentes modelos simulados en este se detallan a continuación (Figura 2, Tablas 1 y 2):

Tabla 1. Dimensiones del ejemplo simulado y mallado de elementos finitos.

\begin{tabular}{|l|l|l|l|}
\hline $\mathrm{N}^{0}$ de plantas: & $4(\mathrm{P} 1, \mathrm{P} 2, \mathrm{P} 3 \mathrm{y}$ P4 $)$ & Espesor muro: & $200 \mathrm{~mm}$ \\
\hline Altura de planta: & $\mathrm{H}=3 \mathrm{~m}$ & Canto de carreras y de zapatas: & $200 \mathrm{~mm}$ \\
\hline Distancia entre pies derechos: & $\mathrm{L}=1,4 / 1,6 / 1,8 \mathrm{~m}$ & Canto de puentes: & $100 \mathrm{~mm}$ \\
\hline Longitud zapata: & $\mathrm{Z}=450 / 600 / 900 \mathrm{~mm}$ & Ancho de pies derechos: & $160 \mathrm{~mm}$ \\
\hline
\end{tabular}

Tabla 2. Valores medios de la madera del entramado y del relleno (8) (10).

\begin{tabular}{|l|c|c|c|c|}
\hline Materiales: & $\mathrm{E}\left[\mathrm{N} / \mathrm{mm}^{2}\right)$ & $\mathrm{G}\left[\mathrm{N} / \mathrm{mm}^{2}\right]$ & C. Poisson & Densidad $\left[\mathrm{kg} / \mathrm{m}^{3}\right]$ \\
\hline Entramado de madera ${ }^{(*)}$ & 12000 & 750 & 0,20 & 800 \\
\hline Relleno del cuartel & 3000 & 1200 & 0,25 & 1800 \\
\hline
\end{tabular}

Carga lineal de los forjados sobre carreras: $27,135 \mathrm{kN} / \mathrm{m}$ (corresponde a una carga de forjado de $3,09 \mathrm{kN} / \mathrm{m}^{2}$ de peso propio y $2,96 \mathrm{kN} / \mathrm{m}^{2}$ de sobrecargas, con una luz de $4,5 \mathrm{~m}$ a cada lado del muro). La carga se ha simulado como continua aplicada directamente sobre las carreras.

${ }^{(*)}$ El material de pies derechos y rellenos se ha considerado como isótropo en aras de simplificar el proceso.
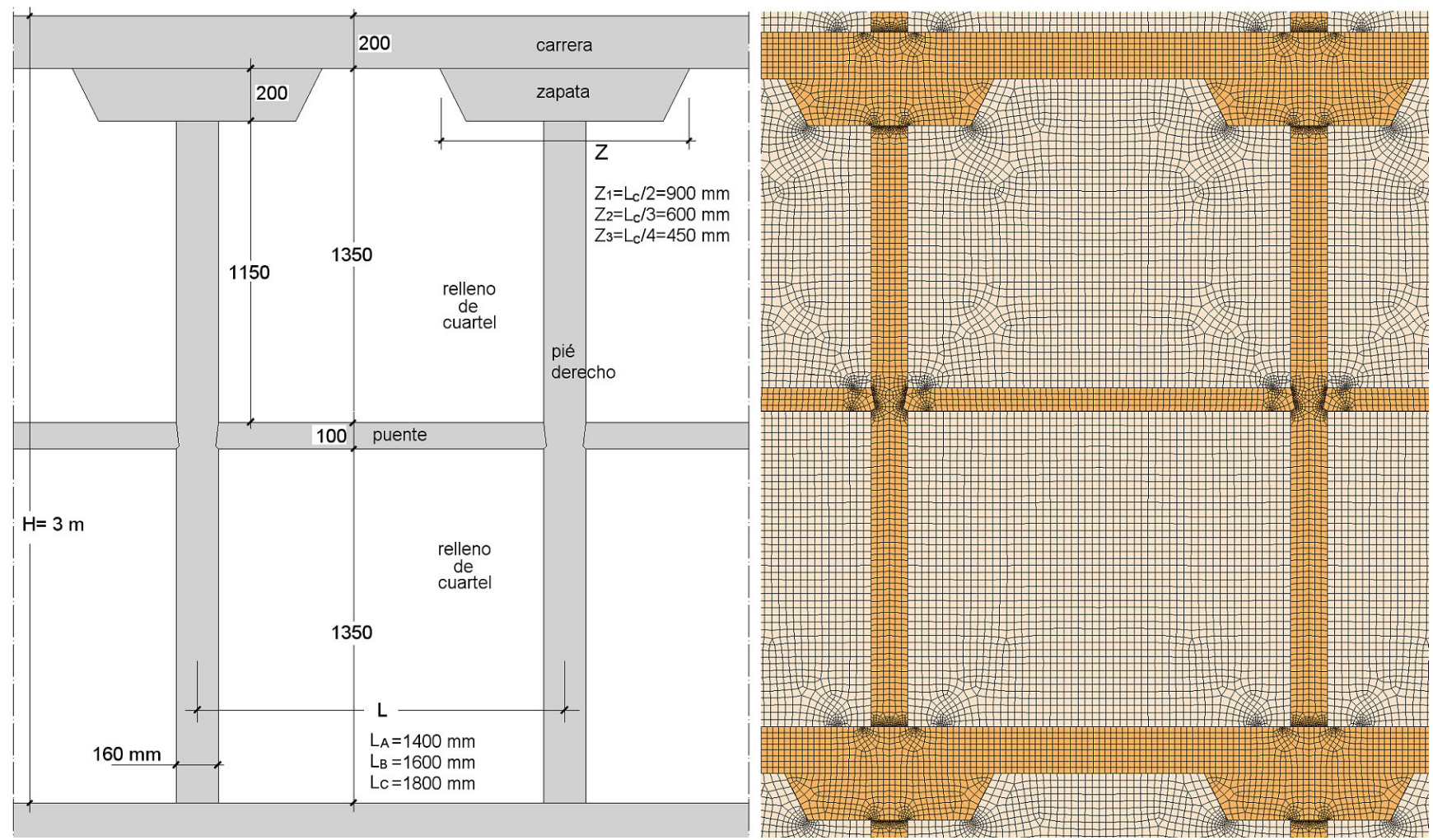

Figura 2. Esquema geometría del muro, y modelo de elementos finitos. 


\section{DESARROLLO}

\subsection{Modelo de comportamiento estructural}

Del análisis de los resultados obtenidos en las diferentes simulaciones, se confirma que la transmisión de cargas gravitatorias se realiza principalmente por compresión vertical entre rellenos de plantas contiguas hasta la cimentación, a través de las carreras que actúan como meros durmientes. Sin embargo, las compresiones de los laterales de los rellenos se desvían a los pies derechos debido a la mayor rigidez a compresión que presentan los pies derechos. Este mismo efecto se observa en la unión de los puentes con los pies derechos, que actúan como puntos de apoyo para el relleno impidiendo el descenso del mismo. Esto, sumado al rozamiento entre relleno y pie derecho, provoca que un porcentaje de la carga que llega a la cabeza de los rellenos se transmita a los pies derechos (Figura 3).

En aquellos casos en los que no existe relleno de los cuarteles inferiores (Figura 3), la acción de los forjados sobre la carrera provoca una deformación (flecha) de la misma siempre mayor que la deformación del relleno superior, produciéndose la separación entre carrera y relleno, formándose un arco de descarga en el material de relleno, sobre los apoyos de la carrera en las zapatas inferiores. De esto se deduce que también en este caso el relleno actúa como trasmisor principal de las cargas gravitatorias frente a la flexión de las carreras, apoyando así el modelo de comportamiento propuesto para el muro ciego.

En la Figura 3 (derecha) se representan las curvas de tensiones verticales en el relleno del mismo cuartel de planta P2 a diferentes alturas. Como se observa, las cargas de la zona lateral del relleno se desvían al puente y la zapata, debido al efecto ménsula antes descrito. La mayor parte de la carga que recibe la zapata se recoge por el pie derecho, pero parte se desvía otra vez al relleno inferior.

\subsection{Análisis del funcionamiento del muro entramado con ausencia total o parcial de cuarteles}

En aquellos edificios en los que no existe relleno en la planta baja (normalmente por reformas posteriores), las tensiones de compresión de los rellenos superiores se canalizan en la segunda planta a los puntos de mayor rigidez, que son las zapatas de los pies derechos, formándose arcos de descarga en combinación con la flexión de las carreras.

Esta situación es muy visible en los entramados de fachada con plantas bajas porticadas: las carreras manifiestan grandes flechas, y los rellenos presentan fisuración siguiendo la forma típica de arco de descarga. Normalmente en estos casos, la excesiva flecha de las carreras está provocada y mantenida por la carga del forjado que apoya directamente sobre la carrera, pero no por los rellenos. Este efecto se acentúa con el fenómeno de fluencia de la madera a lo largo del tiempo, debido al cual las carreras se deforman hasta apoyar en los rellenos inferiores. Sin embargo, dada la dificultad de evaluar dicho fenómeno en estructuras de más de 100 años, la fluencia no se ha incorporado como parámetro de cálculo en este estudio.

En ciertos casos de reformas, al demoler los rellenos de planta baja de forma brusca, se provoca la entrada en carga violenta de las carreras superiores, que pueden llegar a colapsar la estructura.

A continuación se comparan mediante simulación numérica los tres casos siguientes (Figura 4).

La Figura 5 representa los vectores de tensiones principales de compresión en los rellenos de los cuarteles para los casos A y B, quedando patente la formación del arco de descarga cuando no existe relleno del cuartel inferior (caso B).
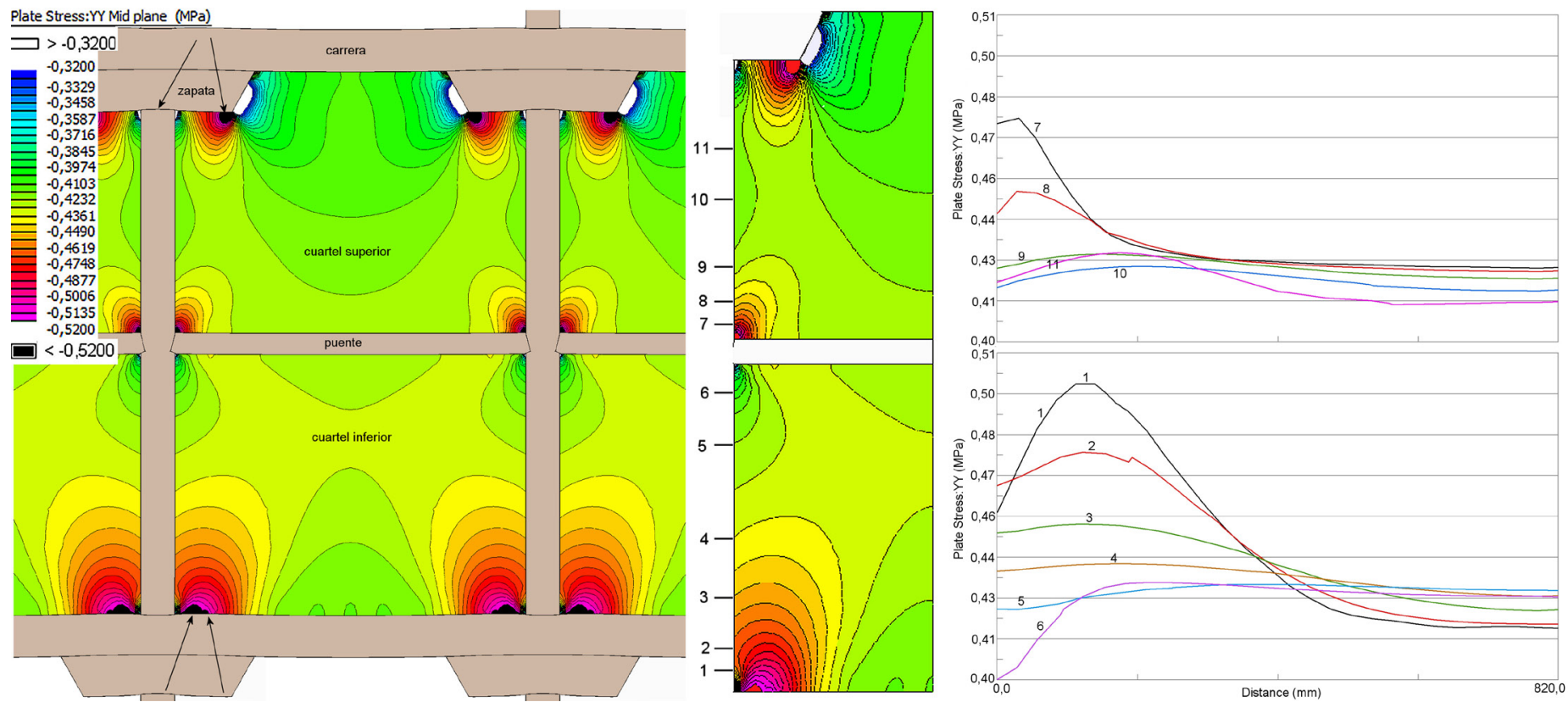

Figura 3. Gráfico (izqda.) y curvas (dcha.) de tensiones verticales en los cuarteles de la planta P2, y efecto de transmisión de cargas del cuartel superior al pie derecho, a través del puente y formación del arco de descarga. 

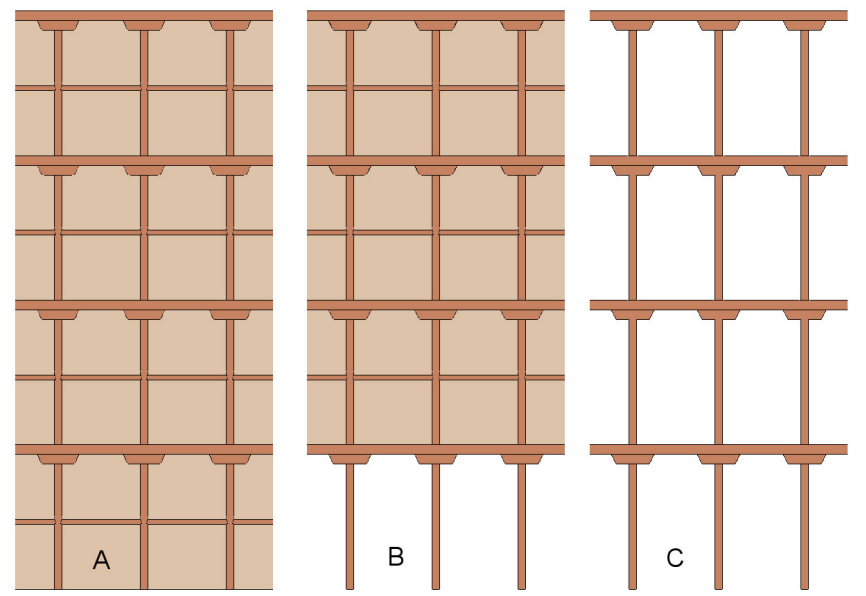

$\mathrm{N}^{\mathrm{o}}$ de plantas:

Altura de planta $(\mathrm{H})$ :

Distancia entre pies derechos (L):

Longitud zapata (Z):

Espesor muro (e):

Canto de carreras y de zapatas:

Canto de puentes:

Ancho de pies derechos (a):

Carga lineal de los forjados sobre carreras:

Materiales: (ver Tabla 2)
4

$3 \mathrm{~m}$

$1800 \mathrm{~mm}$

$\mathrm{L} / 2=900 \mathrm{~mm}$

$200 \mathrm{~mm}$

$200 \mathrm{~mm}$

$100 \mathrm{~mm}$

$160 \mathrm{~mm}$

$27,135 \mathrm{kN} / \mathrm{m}$

Figura 4. Esquema de los tres casos simulados (en las plantas sin cuarteles, se han eliminado las entalladuras de los pies derechos donde embridan los puentes).

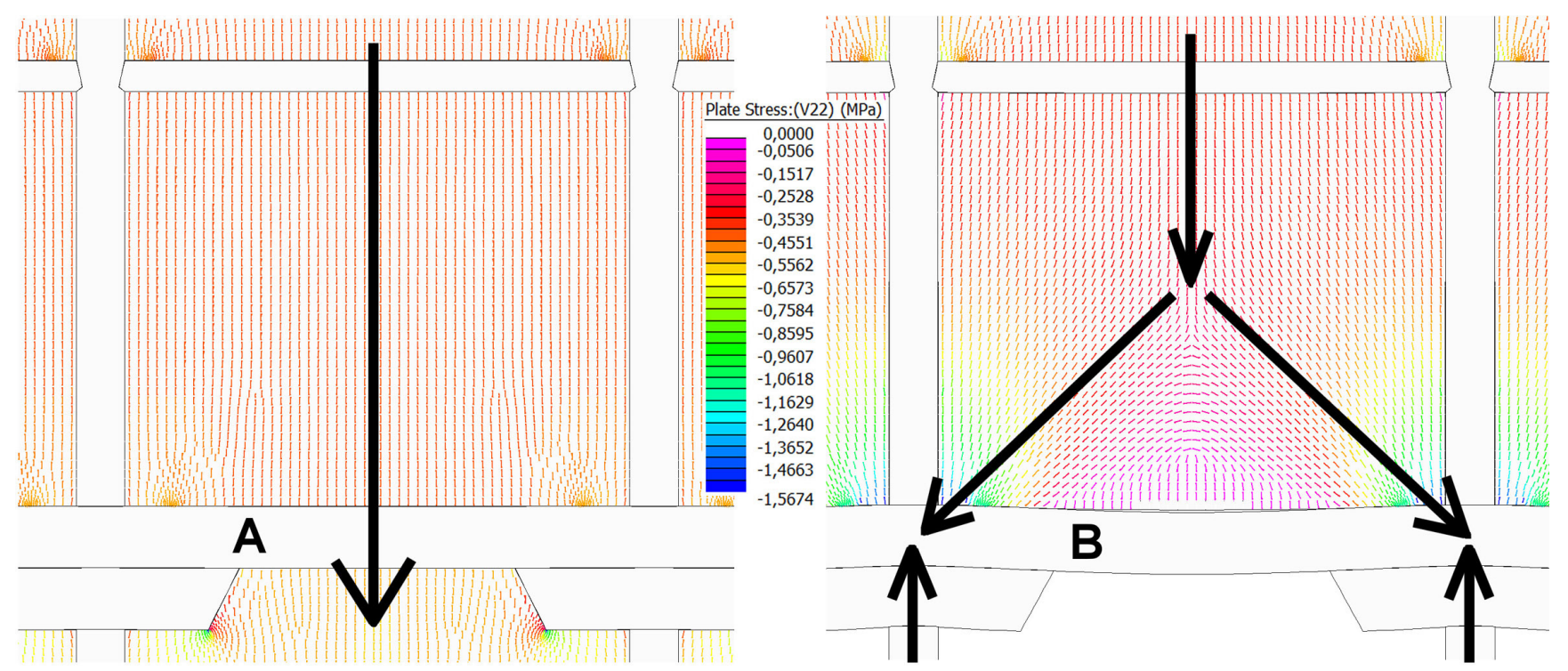

Figura 5. Vectores de tensiones principales de compresión para los casos A y B, en la planta P2.

En el caso A, todos los cuarteles están rellenos, y se evidencian las líneas de compresión vertical hasta la cimentación. En este caso se puede deducir que el relleno está actuando como un muro de carga convencional, sin la colaboración por flexión de las carreras, que funcionan en este caso como meras solerías intermedias, a pesar de que una parte de la carga total se desvía a los pies derechos, tal y como se expuso antes.

En el caso B, se ha suprimido el relleno de la planta P1, operación frecuente en reformas de locales de planta baja, bajo consideraciones equivocadas de la función no estructural de los rellenos. Esta operación provoca que las carreras de techo de dicha planta queden sin el apeo de los rellenos inferiores y entren en carga, produciéndose su flexión debida a la carga del forjado. Como se aprecia en la zona detallada, el relleno de la planta P2 no acompaña la deformación de la carrera en la que apoya (solicitada sobre todo por el peso del forjado), produciéndose un arco de descarga en cuyo vano las tensiones de compresión son casi nulas (color azul oscuro en la Figura 5 dcha.).

En el caso C se han retirado la totalidad de los rellenos, operación no poco frecuente en ciertas rehabilitaciones integra- les en este tipo de edificios. En dicho caso, son las carreras las que absorben por flexión la totalidad de las cargas del forjado.

En la Figura 6 se muestran las curvas de tensión vertical de pies derechos y rellenos en cada planta (a $150 \mathrm{~mm}$ de la base) para los 3 casos. Se evidencia claramente el efecto de arco de descarga en el cuartel de la planta $\mathrm{P} 2$ del caso B.

También es evidente la mayor tensión a la que está sometido el pie derecho de la planta P1 del caso C en comparación al caso A, de lo que se deduce que la retirada de rellenos de un muro entramado, no solo no descarga los pies derechos, sino que aumenta su solicitación, al absorber el $100 \%$ de la carga de los forjados, que en el caso A, se reparten proporcionadamente entre los pies derechos y rellenos.

\section{Estado tensional de la carrera de techo de P1 en los tres casos.}

La Figura 7 representa los valores de tensiones normales a la sección transversal de la viga provocados por su flexión, y la deformada de la carrera de techo de P1 en el entramado, para los tres casos estudiados: 
Caso A: se observan unos valores muy bajos y casi constantes en las tensiones normales de flexión (entre 0,10 y 0,25 $\mathrm{MPa}$ ), lo que indica que casi no existe flexión en la carrera, consecuencia de que está funcionando como una solera entre el relleno superior y el inferior.

Caso B: al desaparecer el relleno inferior, la carrera entra en carga por efecto del peso del forjado, observándose la deformada de flexión y los valores de tensión propios de esta soli- citación (en el vano, compresiones y tracciones máximas en torno a los $2 \mathrm{MPa})$.

Caso C: se han eliminado los rellenos de todas las plantas, por lo que únicamente se tiene en cuenta el peso de los forjados y el peso propio de la madera, que provoca la flexión libre de las carreras. Los valores tensionales en éste caso (en el vano, compresiones y tracciones máximas en torno a los 1,5 $\mathrm{MPa}$ ) son inferiores a los registrados en el caso $\mathrm{B}$, lo que indica que
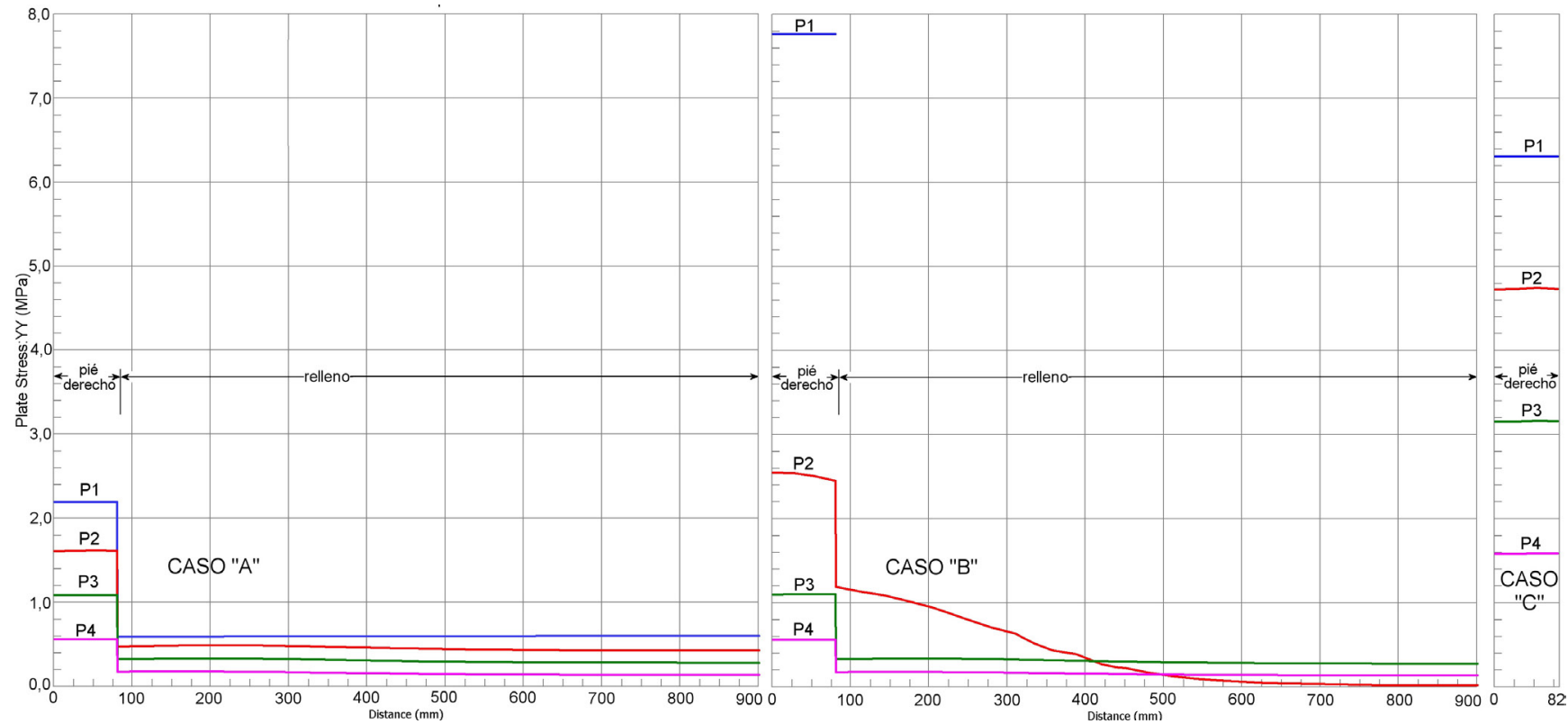

Figura 6. Curvas de tensiones verticales en la base de cada planta para los tres casos (A, B y C).
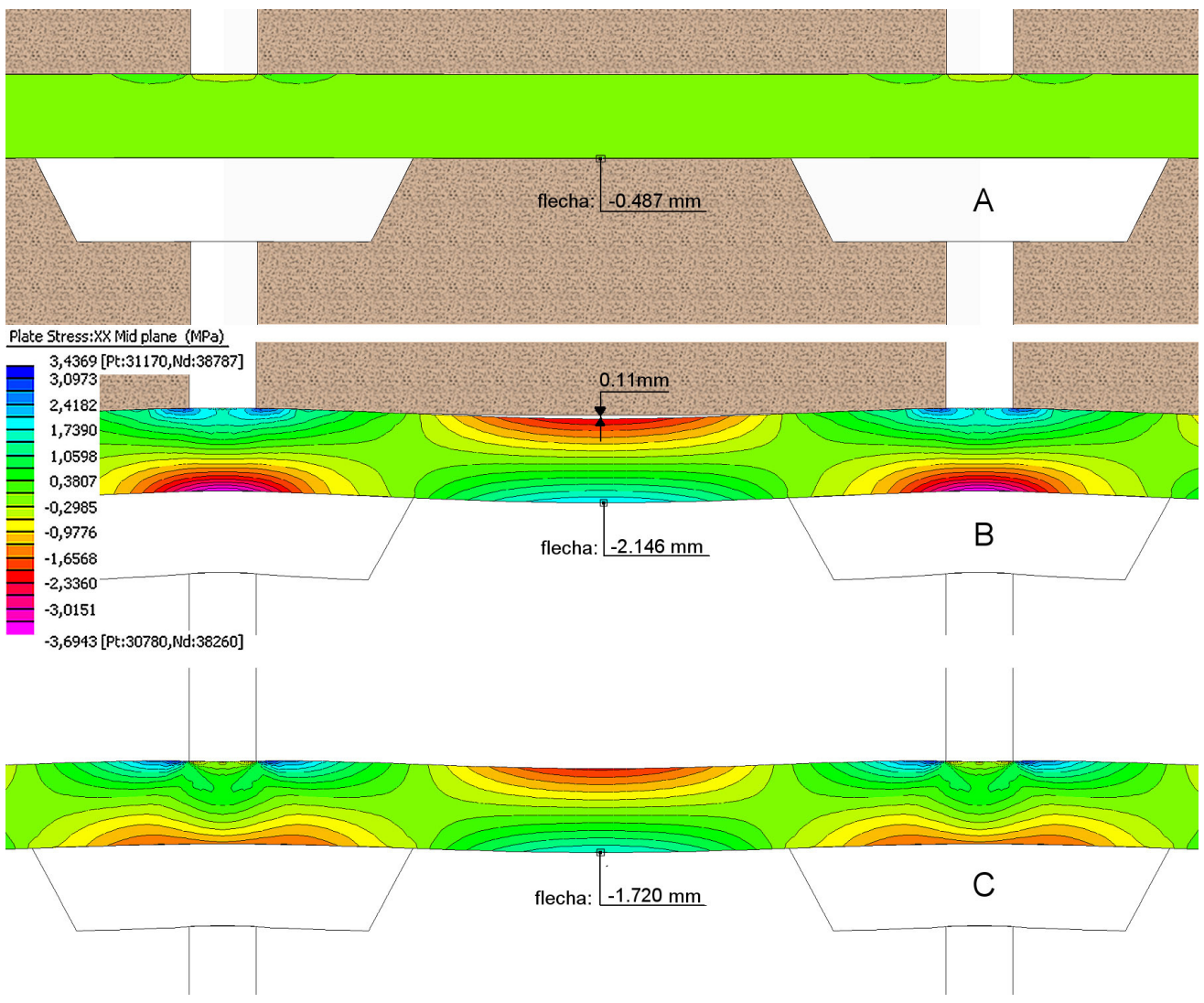

Figura 7. Tensiones normales de flexión y flecha máxima de las carreras de techo de P1, en los tres casos. 
la carrera en dicho caso B está soportando también parte del peso del relleno superior, además del peso del forjado.

En todo este proceso de reparto de pesos, tiene mucha influencia la puesta en carga de la estructura a lo largo de su ejecución. Un pequeño porcentaje de la carga total, aparece con la construcción del entramado, cuando todavía no se han rellenado los cuarteles, y por lo tanto, las carreras tienen una flexión libre. Es este porcentaje de carga el que se absorbe totalmente por las carreras a flexión. Una vez construidos los rellenos, éstos actúan como un apeo natural de las carreras, de tal forma que el resto de cargas (rellenos, tabiquerías, solados, sobrecargas) ya no se transmiten por flexión de las carreras a los pies derechos, sino por compresión directa a través de los rellenos hasta la cimentación.

Sin embargo, a lo largo del tiempo las carreras a flexión adquieren una deformación por fluencia de las fibras de madera, aunque tal deformación nunca se llega a producir de manera plena debido a que la carrera apoya antes en el relleno inferior, que se convierte así en un apeo natural. En estas circunstancias, las cargas que en origen absorbía la carrera por flexión, pasan a circular por compresión al relleno, descargando parcialmente a los pies derechos de su carga inicial.

De todos los casos simulados, se puede deducir que en un muro entramado macizo ( $\sin$ huecos), el pie derecho recibe una parte de la carga del relleno, cuya cuantía está determinada por tres fenómenos distintos que quedan bien representados en la Figura 3:

- El rozamiento entre pie derecho y relleno debido al entomizado de aquel.

- El efecto de apoyo sobre el puente en la zona de su unión con el pie derecho.

- El efecto ménsula de la zapata y carrera.

\subsection{Determinación analítica del reparto de carga entre los pies derechos y los rellenos}

La determinación analítica de la cuantía de dichos efectos en su conjunto, permitirá comprobar el funcionamiento estructural del muro, pero sobre todo, dimensionar los refuerzos necesarios en caso de eliminar un relleno o aumentar la carga total del forjado. Dada la complejidad de dicho análisis, se recurre al método de simulación numérica para establecer los porcentajes aproximados de la carga total que absorben los pies derechos y los rellenos en función de los siguientes parámetros:

- Relación entre los módulos de elasticidad de la madera y rellenos.

- Distancia entre los pies derechos.

- Longitud de la zapata.
Dado el gran número de variables implicadas en la cuantificación del reparto de cargas en un muro de este tipo, se recurre a aproximar en primer término una expresión, en base al parámetro de mayor influencia que es la relación entre módulos de elasticidad de la madera y rellenos $E_{m} / E_{R}$.

En dicha expresión, la única variable es la relación entre los módulos de elasticidad de relleno y postes $\left(E_{m} / E_{R}\right)$. Para las sucesivas comprobaciones, y con el objeto de homogeneizar los resultados, se utiliza como variable la relación entre rigideces a compresión de pies derechos y rellenos $\left(R_{p} / R_{R}\right)$, que a su vez depende de los módulos de elasticidad y de la geometría del cuartel.

Posteriormente, se procede a comprobar el comportamiento de la expresión propuesta aplicándola a otras variables como son la distancia entre pies derechos y la longitud de las zapatas.

Los resultados de estas comprobaciones arrojan valores que se desvían en torno a un $1 \%$ de los obtenidos por la simulación por elementos finitos, por lo que puede considerase suficientemente válida la expresión.

3.3.1. Influencia de la relación entre rigideces a compresión de pies derechos y rellenos en el reparto de cargas

A continuación se estudia la relación entre el cociente de la rigidez a compresión de los pies derechos $\left(\mathrm{R}_{\mathrm{p}}\right)$ y de los rellenos $\left(R_{R}\right)$, y el reparto de cargas del muro en ambos elementos a nivel de la base de cada planta [3]. Las rigidices a compresión $R_{P} y_{R}$ se definen según las ecuaciones [1] y [2] (Figura 8).

$$
\begin{gathered}
R_{p}=(a \times e) E_{m} \\
R_{R}=(b \times e) E_{R} \\
\text { relación rigideces }=\frac{R_{p}}{R_{R}}=\frac{a E_{m}}{b E_{R}}
\end{gathered}
$$

Dado el gran número de variables, se realizan dos supuestos que contemplan de forma independiente la relación $\mathrm{E}_{\mathrm{m}} / \mathrm{E}_{\mathrm{R}} \mathrm{y}$ la relación $\mathrm{a} / \mathrm{b}$, para finalmente englobarlas en una única expresión:

Supuesto «S1»: se estudian tres casos, variando el material de pies derechos y rellenos $\left(\mathrm{E}_{\mathrm{m}} / \mathrm{E}_{\mathrm{R}}\right.$ variable, $\mathrm{a} / \mathrm{b}$ constante).

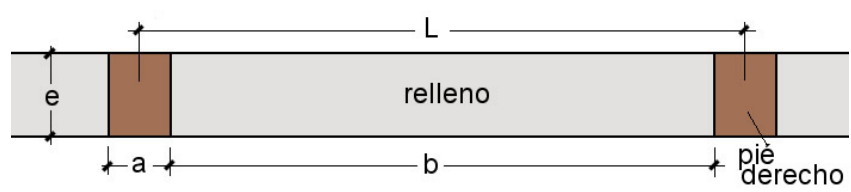

Figura 8. Geometría en planta del muro entramado estudiado.

Tabla 3. Parámetros del supuesto «S1» (Datos madera según CTE para clases resistentes C18, C24 y C35; Datos relleno caso c1: según

\begin{tabular}{|c|c|c|c|c|c|}
\hline \multirow{2}{*}{ Datos fijos: } & $\mathrm{L}$ & zapata $=\mathrm{L} / 2$ & $\mathrm{a}$ & $\mathrm{b}$ & $a / b$ \\
\hline & $1800 \mathrm{~mm}$ & $900 \mathrm{~mm}$ & $160 \mathrm{~mm}$ & $1640 \mathrm{~mm}$ & 0.0975 \\
\hline \multirow{4}{*}{ Datos variables: } & & $\mathrm{E}_{\mathrm{m}}(\mathrm{MPa})$ & $\mathrm{E}_{\mathrm{R}}(\mathrm{MPa})$ & $\mathrm{E}_{\mathrm{m}} / \mathrm{E}_{\mathrm{R}}$ & $\mathrm{R}_{\mathrm{P}} / \mathrm{R}_{\mathrm{R}}$ \\
\hline & Caso c1 & 9000 & 5000 & 1,80 & 0,1756 \\
\hline & Caso c2 & 10000 & 4000 & 2,75 & 0,2683 \\
\hline & Caso c3 & 13000 & 3000 & 4,33 & 0,4227 \\
\hline
\end{tabular}
fábrica de ladrillo macizo de junta delgada y resistencia de mortero fk $=5 \mathrm{MPa}, \rightarrow \mathrm{E}=5000 \mathrm{DB}$ SE-F; Relleno caso c3: relleno base de $\mathrm{E}=3000 \mathrm{MPa}$ (Doudoumis (8), Villanueva (10)); Relleno caso c2: valor medio). 
Supuesto «S2»: se estudian tres casos, variando la distancia entre pies derechos $\left(E_{m} / E_{R}\right.$ constante, $a / b$ variable $)$.

Supuesto «S1»: En primer lugar, se realiza una simulación por elementos finitos de tres casos geométricamente idénti$\cos \left(a / b=\right.$ constante), pero con tres valores diferentes de $\mathrm{E}_{\mathrm{m}} \mathrm{y}$ $\mathrm{E}_{\mathrm{R}}$ (Tabla 3).

De los resultados de la simulación (Tabla 3) se obtienen las curvas de tensiones verticales en pies derechos y rellenos para cada uno de los casos, en las 4 plantas (Figura 9). Los valores de tensiones se miden en una sección horizontal a $150 \mathrm{~mm}$ de la base en cada planta, con el objeto de que las singularidades que se producen en la zona de contacto con la carrera no alteren los resultados:

Se obtienen los valores de carga en pies derechos y rellenos para cada planta en la sección indicada, mediante la integración de las curvas de tensión, y se calculan los porcentajes de carga que recibe el pie derecho respecto del total de carga de pies derechos y rellenos. Los valores de las cargas y los porcentajes obtenidos, ordenados según la relación $\mathrm{R}_{\mathrm{P}} / \mathrm{R}_{\mathrm{R}}$, se muestran en la Tabla 4 .

Como se observa, los porcentajes de reparto no varían significativamente entre plantas, aunque sí con el valor de $R_{P} / R_{R}$ siguiendo una relación polinómica de $2^{\circ}$ grado, por lo que podemos formular una expresión [4] que cuantifique el reparto de carga que absorben los pies derechos (CP) en los casos estudiados:

Porcentaje de carga en pie derecho:

$$
\mathrm{CP}[\%]=-39\left(\frac{a E_{m}}{b E_{R}}\right)^{2}+78\left(\frac{a E_{m}}{b E_{R}}\right)+1,8
$$

$C P$ : porcentaje de carga que recibe el pie derecho (\%) $a, b:$ anchos de pie derecho y relleno [mm]

$E_{m}, E_{R}:$ Módulos de elasticidad de la madera y relleno [MPa]

En la Figura 10 se comparan los resultados obtenidos en la simulación «S1» para los tres casos y cada planta, con la curva proveniente de aplicar la expresión antes propuesta [4] a los valores de $\mathrm{R}_{\mathrm{P}} / \mathrm{R}_{\mathrm{R}}$ empleados en la simulación:

Supuesto «S2»: En segundo lugar, se estudia el comportamiento de la expresión propuesta [4] aplicada a 3 casos variando la separación entre los pies derechos (a/b variable), pero manteniendo el mismo material $\left(\mathrm{E}_{\mathrm{m}} / \mathrm{E}_{\mathrm{R}}=\right.$ constante) (Tabla 5).

Tal y como se procedió en el supuesto «S1», se obtienen las curvas de tensiones verticales en pies derechos y rellenos ob-

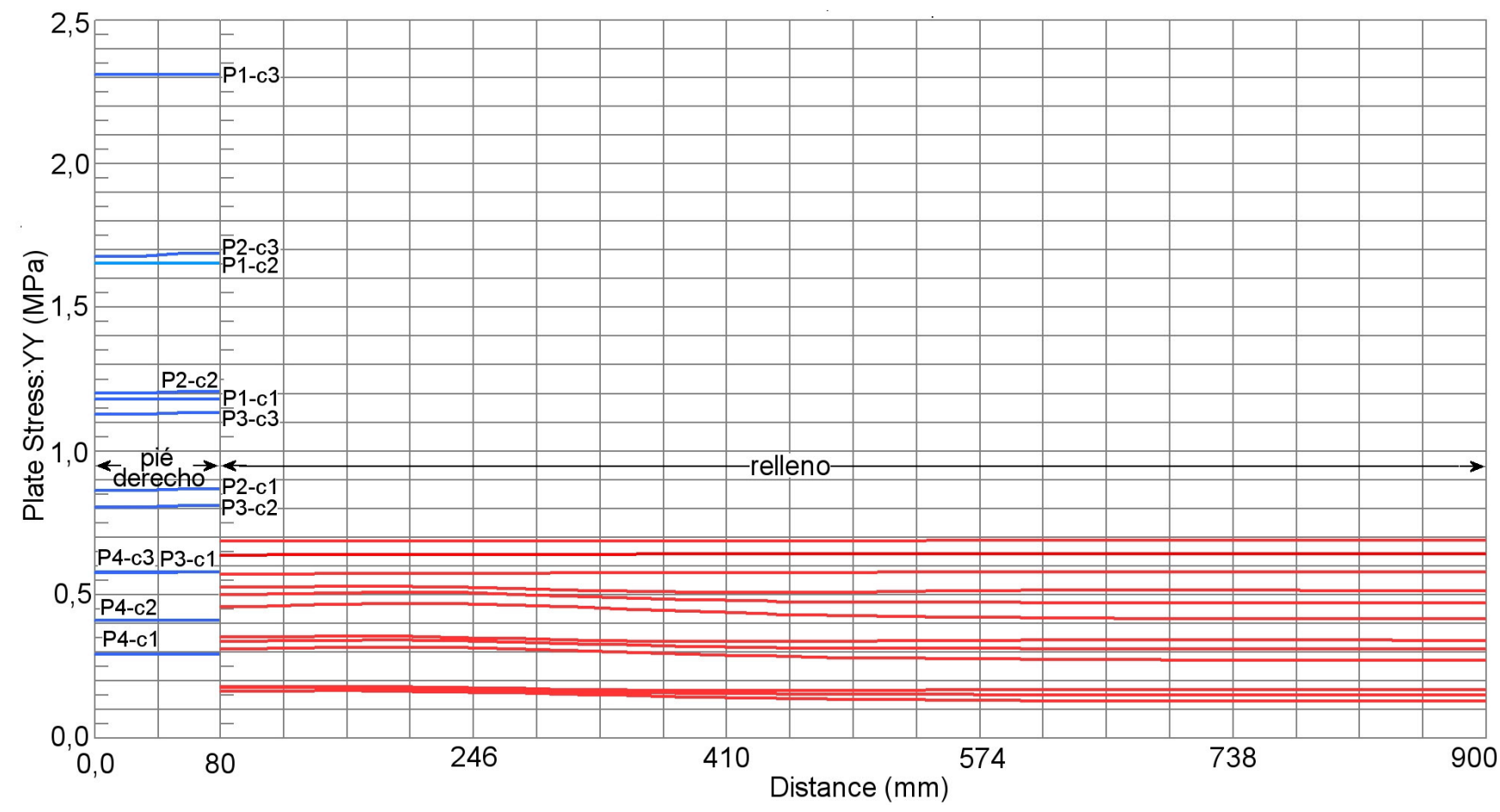

Figura 9. Curvas de tensiones verticales en pies derechos y rellenos en los tres casos del supuesto «S1» (c1, c2 y c3), en una sección horizontal a $150 \mathrm{~mm}$ de la base de cada planta (P1, P2, P3 y P4).

Tabla 4. Valores de carga obtenidos en pies derechos y rellenos en el supuesto «S1».

\begin{tabular}{|l|c|c|c|c|c|c|c|c|c|}
\hline & \multicolumn{3}{|c|}{ Carga en PIE DER. (N/e) } & \multicolumn{2}{c|}{ Carga en RELLENO (N/e) } & \multicolumn{3}{c|}{ \% CARGA PIE DER. (s/tot) } \\
\hline & c1 & c2 & c3 & c1 & c2 & c3 & c1 & c2 & c3 \\
\hline P4 & 23,385 & 32,893 & 46,3 & 139,059 & 129,468 & 116 & 14,40 & 20,26 & 28,53 \\
\hline P3 & 46,413 & 64,74 & 90,597 & 280,716 & 262,269 & 236,304 & 14,19 & 19,80 & 27,71 \\
\hline P2 & 69,385 & 96,505 & 134,801 & 422,43 & 395,151 & 356,719 & 14,11 & 19,63 & 27,43 \\
\hline P1 & 94,514 & 132,347 & 184,783 & 562,713 & 524,904 & 472,079 & 14,38 & 20,14 & 28,13 \\
\hline \multicolumn{9}{|c|}{} & \multicolumn{9}{|c|}{ Media $(\%):$} & $\mathbf{1 4 , 2 7}$ & $\mathbf{1 9 , 9 6}$ & $\mathbf{2 7 , 9 5}$ \\
\hline
\end{tabular}

\begin{tabular}{c|c|c|c|}
\multirow{2}{*}{ Para $\mathrm{R}_{\mathrm{p}} / \mathrm{R}_{\mathrm{R}}=$} & $\mathbf{c 1}$ & $\mathbf{c 2}$ & $\mathbf{c 3}$ \\
\cline { 2 - 4 } & 0,1756 & 0,2683 & 0,4227 \\
\hline
\end{tabular}


Tabla 5. Parámetros del supuesto «S2».

\begin{tabular}{|l|c|c|c|c|c|}
\hline \multirow{3}{*}{ Datos fijos: } & $\mathrm{E}_{\mathrm{m}}(\mathrm{MPa})$ & $\mathrm{E}_{\mathrm{R}}(\mathrm{MPa})$ & $\mathrm{E}_{\mathrm{m}} / \mathrm{E}_{\mathrm{R}}$ & $\mathrm{a}$ & \multirow{2}{*}{} \\
\cline { 2 - 6 } & 12000 & 3000 & 4,0 & $160 \mathrm{~mm}$ & \\
\hline \multirow{4}{*}{ Datos variables: } & & $\mathrm{L}(\mathrm{mm})$ & zapata=L/2 $(\mathrm{mm})$ & $\mathrm{b}(\mathrm{mm})$ & $\mathrm{R}_{\mathrm{P}} / \mathrm{R}_{\mathrm{R}}$ \\
\cline { 2 - 6 } & Caso c1 & 1400 & 700 & 1240 & 0,5161 \\
\cline { 2 - 6 } & Caso c2 & 1600 & 800 & 1440 & 0,4444 \\
\cline { 2 - 6 } & Caso c3 & 1800 & 900 & 1640 & 0,3902 \\
\hline
\end{tabular}

Tabla 6. Valores de carga obtenidos en pies derechos y rellenos en el supuesto «S2».

\begin{tabular}{|l|c|c|c|c|c|c|c|c|c|}
\hline & \multicolumn{3}{|c|}{ Carga en PIE DER. (N/e) } & \multicolumn{2}{c|}{ Carga en RELLENO (N/e) } & \multicolumn{3}{c|}{ \% CARGA PIE DER. (s/tot) } \\
\hline & c1 & c2 & c3 & c1 & c2 & c3 & c1 & c2 & c3 \\
\hline P4 & 41,29 & 42,81 & 44,05 & 84,63 & 100,96 & 117,87 & 32,79 & 29,78 & 27,20 \\
\hline P3 & 81,14 & 83,94 & 86,23 & 172,61 & 206,53 & 240,96 & 31,98 & 28,90 & 26,35 \\
\hline P2 & 120,97 & 125 & 128,35 & 260,6 & 312,49 & 363,65 & 31,70 & 28,57 & 26,09 \\
\hline P1 & 165,63 & 170,75 & 175,03 & 344,03 & 412,66 & 481,63 & 32,50 & 29,27 & 26,65 \\
\hline \multicolumn{9}{|r|}{} \\
\hline \multicolumn{9}{|r|}{}
\end{tabular}

\begin{tabular}{|c|c|c|c|}
\multirow{2}{*}{$\operatorname{Para} L(\mathrm{~mm})=$} & $\mathbf{c 1}$ & $\mathbf{c 2}$ & $\mathbf{c 3}$ \\
\cline { 2 - 4 } & 1400 & 1600 & 1800 \\
\hline
\end{tabular}

tenidas en la simulación para cada caso y planta, y sobre ellas, se obtienen los valores de carga mediante la integración de las curvas de tensiones, calculándose los porcentajes de carga que recibe el pie derecho sobre el total de carga de pies derechos y rellenos. Los valores de las cargas y los porcentajes obtenidos, ordenados según la relación $\mathrm{R}_{\mathrm{P}} / \mathrm{R}_{\mathrm{R}}$, se muestran en la Tabla 6 .

En la Figura 11 se comparan los resultados obtenidos en la simulación «S2» para los tres casos y cada planta, con la curva resultado de aplicar la expresión antes propuesta a los valores de $R_{\mathrm{P}} / \mathrm{R}_{\mathrm{R}}$ empleados en la simulación.

En dicha figura se observa que la expresión antes propuesta, se ajusta suficientemente a los datos obtenidos en esta simulación (sobre todo en las plantas P1 y P2) con desviaciones máximas de un $1,1 \%$ para la planta $\mathrm{P} 4$, de lo que se deduce su validez dentro de los rangos de separación entre pies derechos (L) usuales en este tipo de edificaciones.

3.3.2. Influencia de la longitud de la zapata $L_{z}$ en el reparto de cargas

A continuación se estudia la relación entre la longitud de la zapata $Z$ (en términos de $L / Z$, siendo $L$ la separación entre

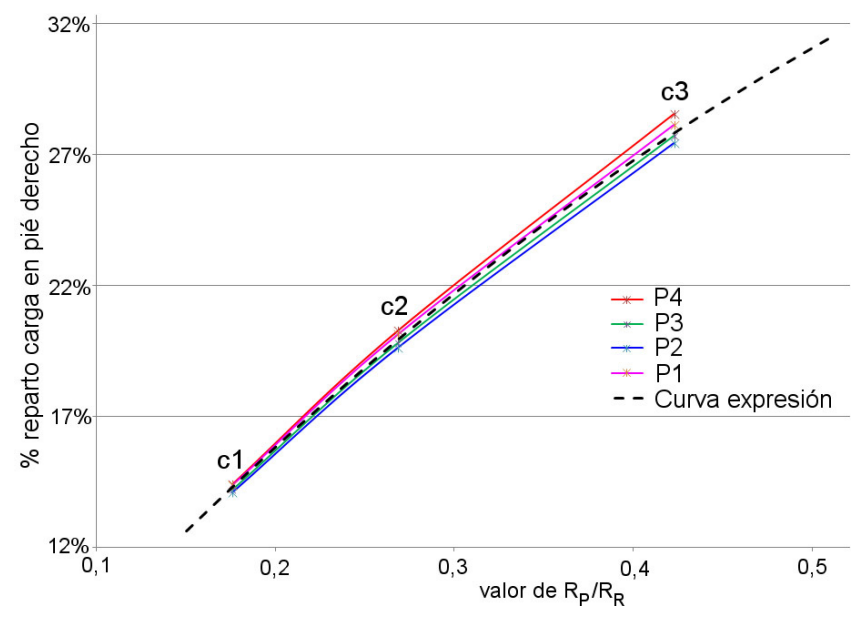

Figura 10. Ajuste de la expresión propuesta a los datos del supuesto «S1». pies derechos) y la cuantía de carga que se transfiere del relleno y carrera al pie derecho en la base de cada planta. Para ello se recurre a simular por elementos finitos tres casos con idéntica separación entre pies derechos y diferentes longitudes de zapata, y con idéntico material de pies derechos y rellenos (Tabla 7).

Del mismo modo que en el apartado anterior, de los resultados de la simulación se obtienen las curvas de tensiones verticales en pies derechos y rellenos para cada uno de los casos, en las 4 plantas. Los valores de tensiones se miden en una sección horizontal a $150 \mathrm{~mm}$ de la base en cada planta.

Sobre dichas curvas de tensiones, se obtienen los valores de carga en pies derechos y rellenos para cada planta en la sección indicada, mediante la integración de las curvas, y se calculan los porcentajes de carga que recibe el pie derecho sobre el total de carga de pies derechos y rellenos. Los valores de las cargas y los porcentajes obtenidos, ordenados según el valor de Z, se muestran en la Tabla 8.

De los resultados obtenidos, se deduce que no existen variaciones significativas de las tensiones en pies derechos y rellenos debidas a las diferentes longitudes de zapata.

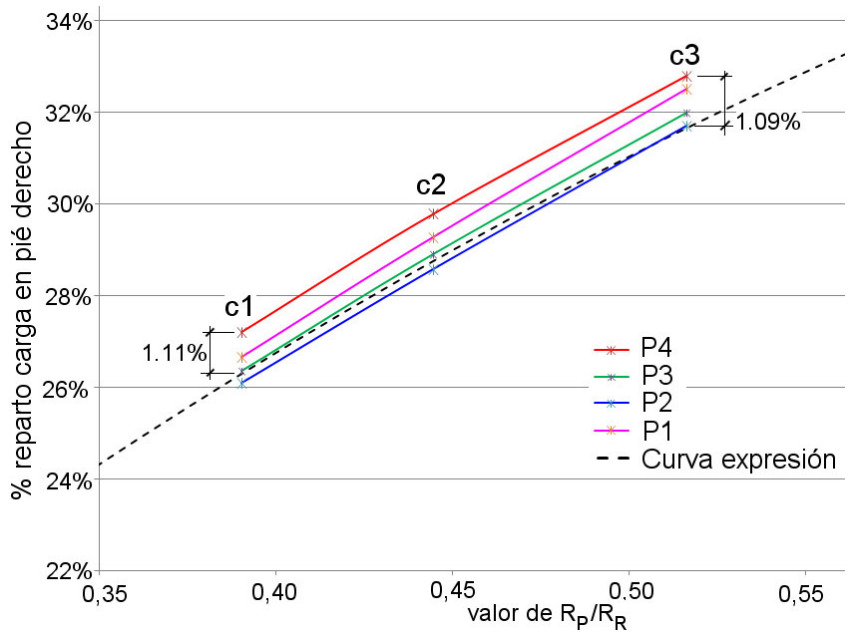

Figura 11. Comparación de la expresión propuesta con los datos del supuesto «S2». 
Tabla 7. Parámetros de la simulación para distintas longitudes de zapata.

\begin{tabular}{|c|c|c|c|c|c|c|c|}
\hline \multirow{2}{*}{$\begin{array}{l}\text { Datos variables: } \\
\text { Z (long. zapata)= }\end{array}$} & Caso c1 & Caso c2 & Caso c3 & \multirow{2}{*}{\multicolumn{4}{|c|}{ (para $L=1800 \mathrm{~mm}$ ) }} \\
\hline & $\mathrm{L} / 4=450 \mathrm{~mm}$ & $\mathrm{~L} / 3=600 \mathrm{~mm}$ & $\mathrm{~L} / 2=900 \mathrm{~mm}$ & & & & \\
\hline \multirow{2}{*}{ Datos fijos: } & $\mathrm{E}_{\mathrm{m}}(\mathrm{MPa})$ & $\mathrm{E}_{\mathrm{R}}(\mathrm{MPa})$ & $\mathrm{E}_{\mathrm{m}} / \mathrm{E}_{\mathrm{R}}$ & $\mathrm{a}$ & $\mathrm{b}$ & $\mathrm{a} / \mathrm{b}$ & $\mathrm{R}_{\mathrm{p}} / \mathrm{R}_{\mathrm{R}}$ \\
\hline & 12000 & 3000 & 4,0 & $160 \mathrm{~mm}$ & 1640 & 0,09756 & 0,3902 \\
\hline
\end{tabular}

Tabla 8. Valores de carga obtenidos en pies derechos y rellenos en la simulación con diferentes longitudes de zapata.

\begin{tabular}{|c|c|c|c|c|c|c|c|c|c|}
\hline & \multicolumn{3}{|c|}{ Carga en PIE DER. (N/e) } & \multicolumn{3}{|c|}{ Carga en RELLENO (N/e) } & \multicolumn{3}{|c|}{ \% CARGA PIE DER. (s/tot) } \\
\hline & c1 & c2 & c3 & c1 & c2 & c3 & c1 & c2 & c3 \\
\hline $\mathrm{P}_{4}$ & 43,834 & 44,031 & 43,702 & 118,89 & 118,55 & 118,59 & 26,94 & 27,08 & 26,93 \\
\hline $\mathrm{P}_{3}$ & 85,712 & 86,076 & 85,543 & 242,017 & 241,378 & 241,369 & 26,15 & 26,29 & 26,17 \\
\hline $\mathrm{P} 2$ & 127,952 & 128,492 & 127,786 & 364,40 & 363,446 & 363,361 & 25,99 & 26,12 & 26,02 \\
\hline $\mathrm{P} 1$ & 175,176 & 175,00 & 174,715 & 483,102 & 482,804 & 482,139 & 26,61 & 26,60 & 26,60 \\
\hline \multicolumn{7}{|r|}{ Media(\%): } & 26,42 & 26,52 & 26,43 \\
\hline
\end{tabular}

$\operatorname{Para} \mathrm{Z}[\mathrm{mm}]=$

\begin{tabular}{|c|c|c|}
\hline c1 & c2 & c3 \\
\hline $\mathrm{L} / 4$ & $\mathrm{~L} / 3$ & $\mathrm{~L} / 2$ \\
\hline 450 & 600 & 900 \\
\hline
\end{tabular}

Por lo tanto, el efecto de ménsula se localiza en el entorno próximo al pie derecho, y la longitud de la zapata apenas influye en la transmisión de cargas del relleno al pie derecho.

En la Figura 12 se comparan los resultados obtenidos en la simulación para los tres casos y cada planta, con la curva resultado de aplicar la expresión antes propuesta al valor de $\mathrm{R}_{\mathrm{p}}$ / $R_{R}$, que en este caso es constante $\left(R_{P} / R_{R}=0,2650\right)$ :

Se observa que la expresión propuesta se adapta mejor a los resultados de las plantas $\mathrm{P}_{2}$ y $\mathrm{P}_{3}$, desviándose el valor hasta un $0,64 \%$ en el caso de la planta P1.

Una vez obtenido el valor de $\mathrm{CP}$, se puede obtener el valor medio de la tensión en un pie derecho $\left(\sigma_{p}\right)$ de una planta mediante la siguiente expresión [5]:

$$
\sigma_{P}=C P[\%] \frac{Q \times L}{S_{P} \times 100}\left[\frac{N}{m^{2}}\right]
$$

$Q$ : carga lineal estimada total que llega a la base de la planta $[\mathrm{N} / \mathrm{mm}]$ $L$ : distancia entre pies derechos [mm]

$S_{P}$ : área de la sección del pie derecho $\left[\mathrm{mm}^{2}\right]$.

\section{CONCLUSIONES}

En el caso de muros entramados ciegos, el comportamiento estructural es similar al de un muro compuesto, transmitiéndose las cargas gravitatorias (peso propio y forjados) a la cimentación a través de los pies derechos de madera y los rellenos siguiendo porcentajes en función de las rigideces a compresión de ambos componentes.

Del estudio realizado, se puede concluir que dado el pequeño margen de error entre los valores de la expresión propuesta y los resultados de las diferentes simulaciones, podemos aceptar dicha expresión [6] como válida para determinar la cuantía de carga CP\% que reciben los pies derechos de un entramado en la base de cada planta, dentro de los límites establecidos en el presente estudio, y para muros entramados ciegos con puentes «a medias».

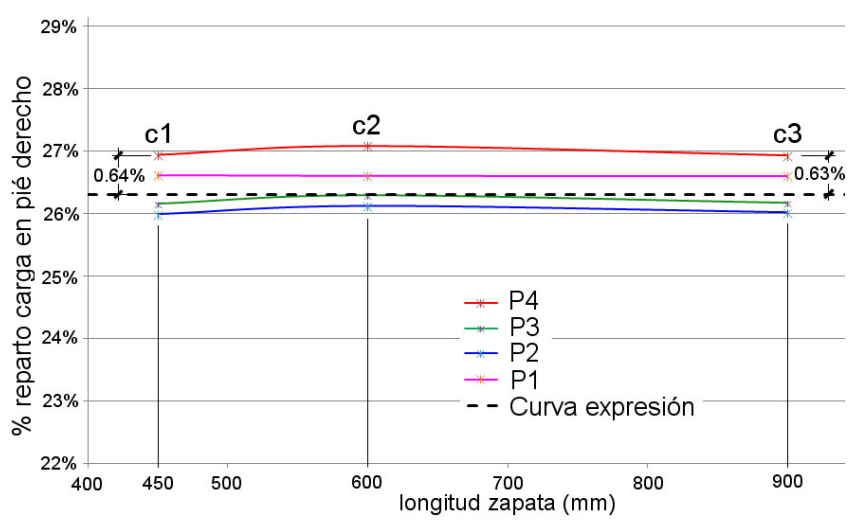

Figura 12. Ajuste de la expresión propuesta a los datos de la simulación.

$$
C P[\%]=-39\left(\frac{a E_{m}}{b E_{R}}\right)^{2}+78\left(\frac{a E_{m}}{b E_{R}}\right)+1,8
$$

$a, b$ : anchos de pie derecho y relleno [mm]

$E_{m}, E_{R}$ : Módulos de elasticidad de la madera y relleno [MPa]

Límites de validez de la expresión:

Distancia entre pies derechos $a+b[\mathrm{~mm}]: 1400 \leq \mathrm{L} \leq 1800$

Longitud de zapata [mm]: $450 \leq \mathrm{Z} \leq 900$

Mód elasticidad madera $\left[\mathrm{N} / \mathrm{mm}^{2}\right]: \quad 9000 \leq \mathrm{E}_{\mathrm{m}} \leq 13000$

Mód elasticidad relleno $\left[\mathrm{N} / \mathrm{mm}^{2}\right]: \quad 3000 \leq \mathrm{E}_{\mathrm{R}} \leq 5000$

En el caso de muros con ausencia de rellenos, el mecanismo de transferencia de cargas se basa en la formación de un arco de descarga en el relleno sobre el hueco, que deriva dichas cargas directamente a los pies derechos inferiores, liberando así a la carrera que únicamente soporta la carga del forjado que apoya en ella.

La cuantificación del estado tensional de los componentes del muro entramado permitirá al técnico evaluar la necesidad de reforzarlo, así como optimizar el diseño y dimensionamiento de dicho refuerzo. 


\section{REFERENCIAS}

(1) González-Redondo, E., Aroca-Hernández-Ros, R. (2005). Construcción de una casa con entramado de madera en Madrid en 1759. En $4^{\circ}$ Congreso nacional de historia de la construcción .Cádiz: COAAT.

(2) Lozano-Apolo, G., Lozano-Martínez-Luengas, A. (1995). Curso de técnicas de intervención en el patrimonio arquitectónico. Tomo I: reestructuración en madera. Gijón: Ed. Consultores Técnicos de la Construcción CB.

(3) Santa Cruz-Astorqui, J. (2012). Estudio tipológico, constructivo y estructural de las casas de corredor en Madrid (Tesis Doctoral). Madrid: Universidad Politécnica de Madrid. http://oa.upm.es/14326/.

(4) Cardoso, R, Lopes, M., Bento, R. (2004, 1-6 de agosto). Seismic assessment of 'Pombalino' buildings. En 13th World Conference on Earthquake EngineerinG. Paper No. 925. Vancouver, B.C., Canada.

(5) Mendes, N., Lourenço, P.B., Costa, A.C. (2010, 11-15 de octubre). Seismic vulnerability of ancient masonry buildings. En Semana de Engenharia 2010. Guimaraes.

(6) Ramos, L.F., Lourenço, P.B. (2004). Modeling and vulnerability of historical city centers in seismic areas: a case study in Lisbon. Engineering Structures, 26(9): 1295-1310, doi: http://dx.doi.org/10.1016/j.engstruct.2004.04.008.

(7) Biegholdt, H.A., Thiele, R. (1999). Timber-masonry composite structures - a bearing structure model. En Brebbia, C.A. (Ed.) Structural Studies, Repairs and Maintenance of Historical Buildings VI, The Built Environment, vol. 42. United Kingdom: Wessex Institute of Technology, Alemania: W. JAGER Technical University of Dresden.

(8) Doudoumis I. N. (2010). Analytical modelling of traditional composite timber-masonry walls. Advanced Material Research, 133-134: 441-446, doi: http://dx.doi.org/10.4028/www.scientific.net/AMR.133-134.441.

(9) Polensek, A. (1976). Finite Element Analysis of Wood-Stud Walls. Journal of the Structural Division, 102(7): $1317-1335$.

(10) Villanueva-Domínguez, L., García-Santos, A. (2001). Manual del yeso. Madrid: CIE Inversiones Editoriales Dossat-2000, S.L. 